\title{
Predicting the Performance of Different Fluidized and Spouted Beds for Spray Granulation using CFD-DEM Simulations
}

Paul Kieckhefen ${ }^{1}$, Moritz Höfert'2, Swantje Pietsch'1, Stefan Heinrich ${ }^{1}$

1 Institute of Solids Process Engineering and Particle Technology, Hamburg University of Technology, Germany

BASF SE, Ludwigshafen am Rhein, Germany

\section{Introduction}

Fluidized beds are excellent apparatuses for the formation of tailor-made particles

Granulation: layered growth of particles by spraying solids-containing liquid

Microprocesses in droplets and on surface liquid determine structure of particle and therefore its properties

CFD-DEM simulations provide detailed insight into hydrodynamic behavior of fluidized beds

- Evaporation of surface liquid, droplet motion and deposition can be tracked for every particle

Track fate of individual particles

Track properties of droplets and impact parameters

- Goal: Predict particle structure from simulations directly

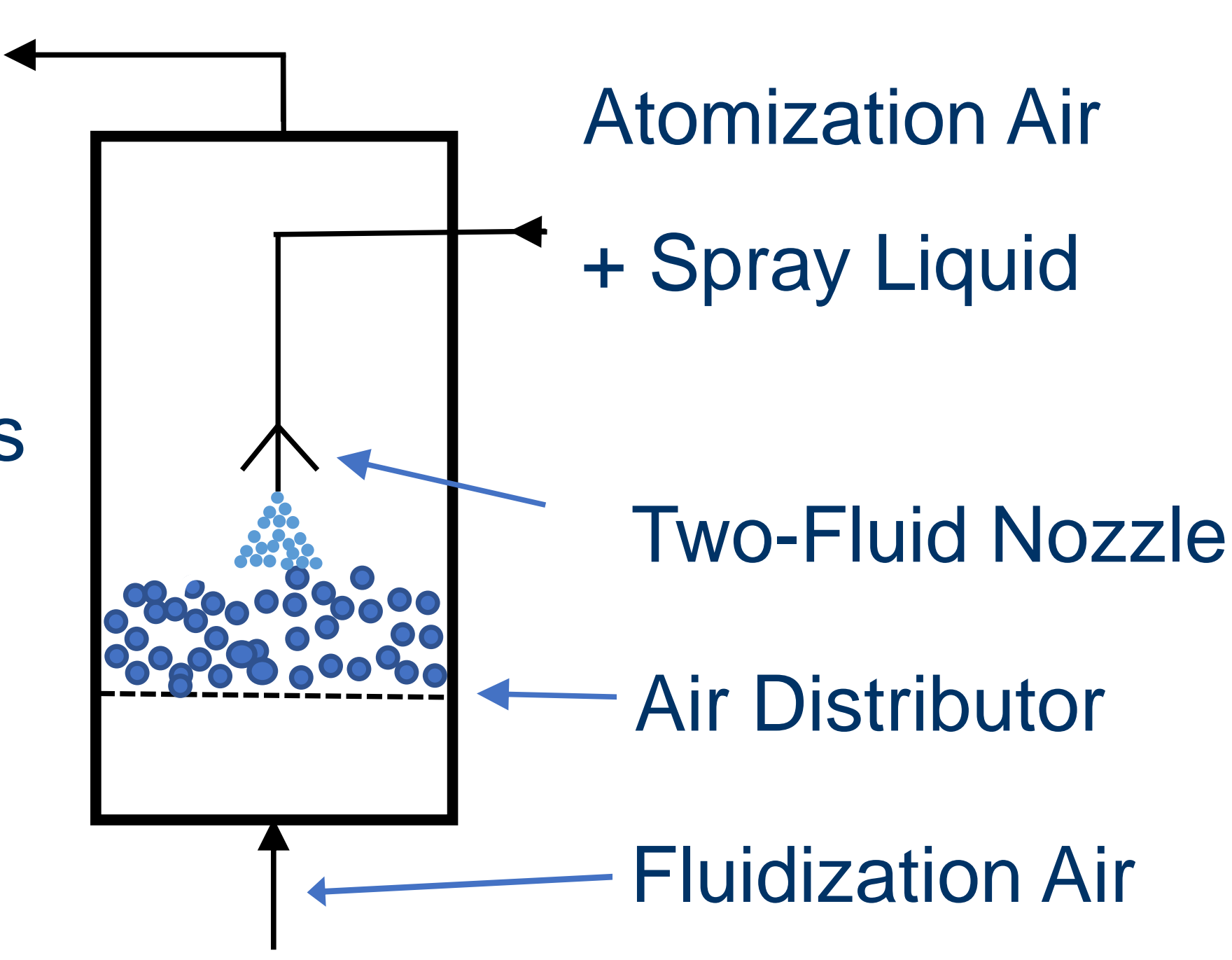

Schematic of a Fluidized Bed Spray Granulation Process
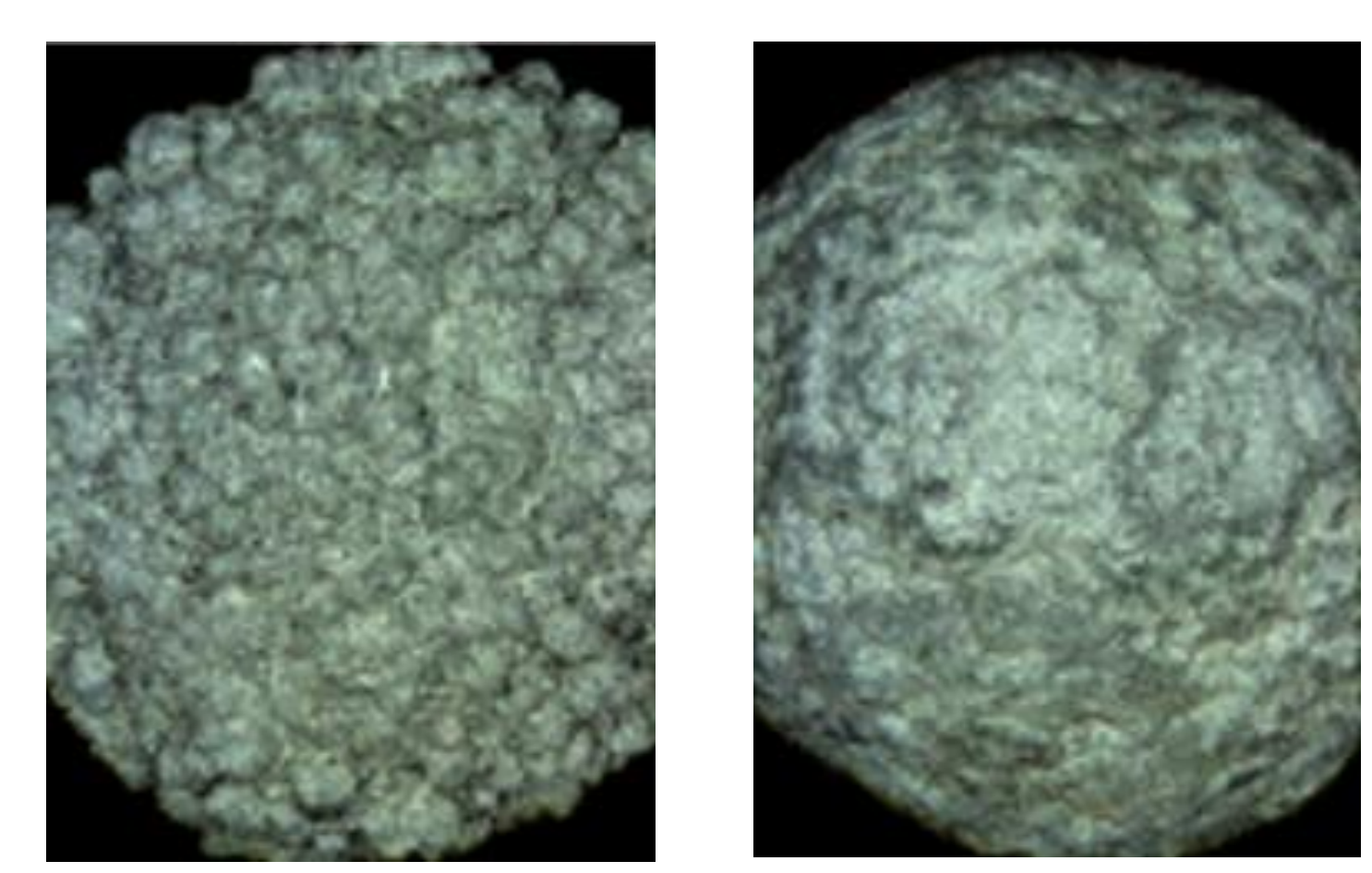

Granulation Products exhibiting Different Surface Structures
Microprocesses in Layering Spray Granulation

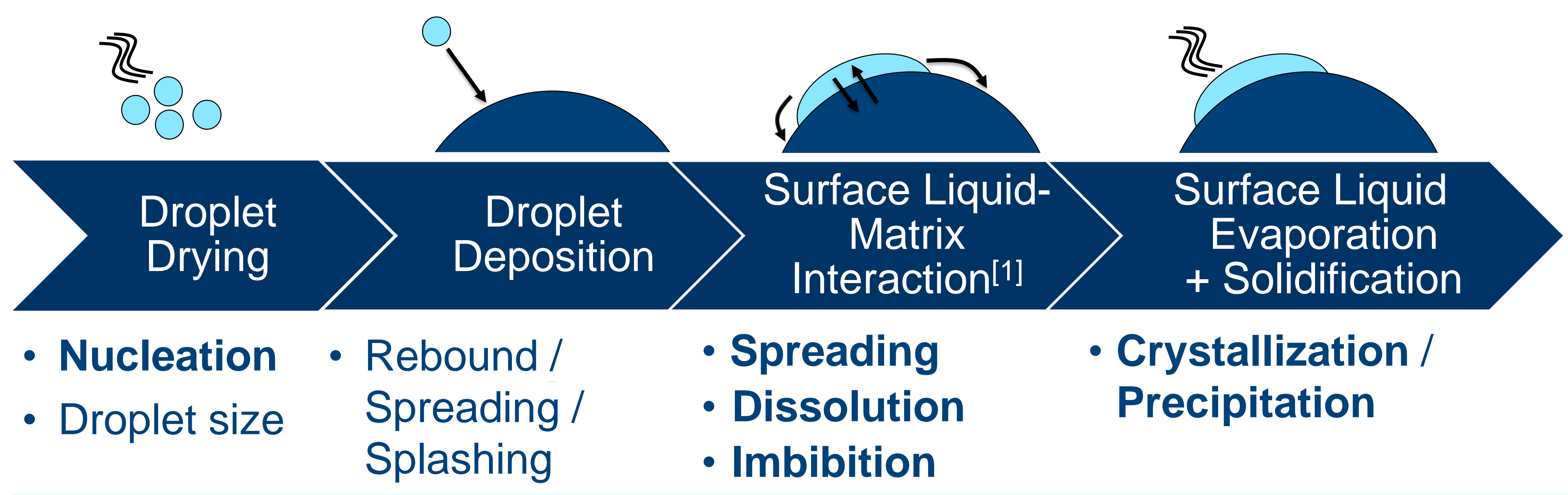

Tracking of impacted droplet state and timescale of drying can be used as target variables/tracked quantities in lieu of resolving surface processes

\section{Workflow for Predicting Product Properties}

Prior State of the Art $[2,3]$

\section{Calibration}

Process Conditions $\downarrow$ Experiment

Product Property

\section{Mapping Process Conditions}

Scalar Drying Potential $\eta_{\text {drying }}=1-\frac{y_{\mathrm{H}_{2} \mathrm{O}}^{\mathrm{wb}}-y_{\mathrm{H}_{2} \mathrm{O}}^{\text {out }}}{y_{\mathrm{H}_{2} \mathrm{O}}^{\mathrm{wb}}-y_{\mathrm{H}_{2} \mathrm{O}}^{\mathrm{in}}}$ Product Property $=\mathrm{f}\left(\eta_{\text {drying }}\right)$

\section{Prediction}

\section{New Process Conditions} $\downarrow$ Apply Mapping Predicted Product Properties

Easy

Usable for salt solutions Unusable for suspensions Restricted to similar geometry
Our Approach

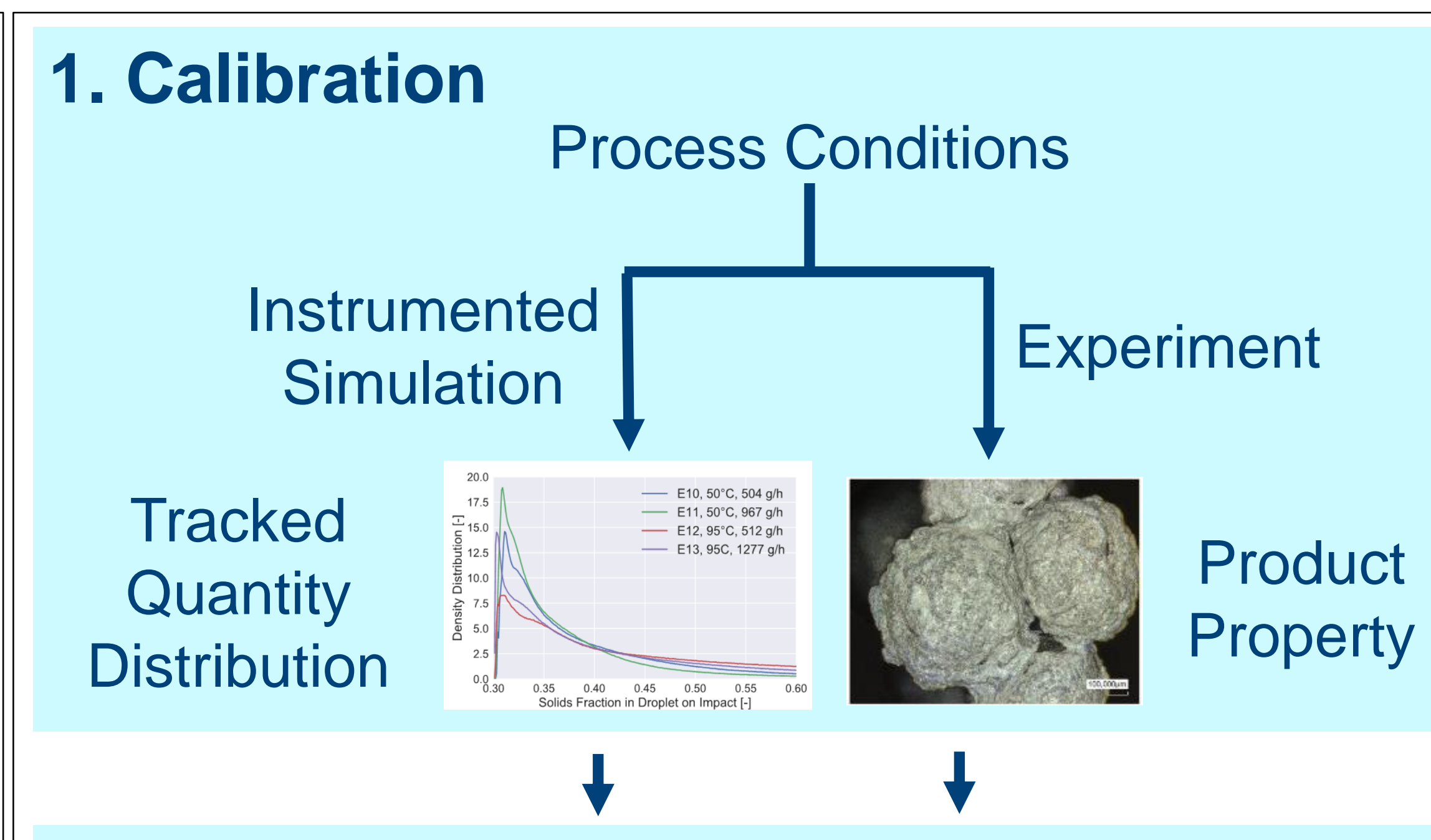

2. Mapping Product Property $=f($ Moments of Tracked Quantity Distribution)

\section{Prediction}

$$
\begin{aligned}
& \text { Different Process Simulation } \longrightarrow \text { Predicted Product } \\
& \begin{array}{lll}
\text { Geometry } & \text { Application } & \text { Properties }
\end{array}
\end{aligned}
$$

+ Geometry-independent + Usable for suspension + Usable for salt solutions + Wider applicability Operates on distributions - Requires simulations of particles

Our concept uses an indirect approach in relating product properties to quantifiers of the microprocesses to their resulting surface structures and thus particle properties. This allows for

Prediction of Scale-Up Effects (incl. dissimilar proportions)

Diagnostics in Case of Sub-Par Product Quality

\section{References}

] Heine et al.: Droplet deposition on amorphous particles in a fluidized bed spray agglomeration process, Granulation Workshop, Lausanne, (2013).

Schmidt et al.: Shell porosity in spray fluidized bed coating with suspensions,

Advanced Powder Technology (2017).

[3] Rieck et al.: Influence of drying conditions on layer porosity in fluidized bed spray granulation Powder Technology (2015)

\section{Product Property - Tracked Quantity Mapping}

Granulation experiments in GF3 (ø $250 \mathrm{~mm}$ )

bottom-spray fluidized bed, 31 total experiments

- Vary spray rate, air temperature, spray air temperature, spray pressure (droplet size)

- Injection of sodium benzoate (30 wt-\%) onto crystalline cellulose particles $(d=650 \mu \mathrm{m})$

Surface roughness characterization using confocal laser-scanning microscope (Keyence)

Digital twin simulations with same process conditions, tracking

\section{- Particle liquid layer evaporation time $t_{\text {evap }}$}

- Droplet solution concentration $x_{\text {s,impact }}$

- Droplet impact velocity $v_{\text {impact }}$

Perform linear regression between statistical moments $\mu$ of tracked quantities in sim. and roughness values $R_{\Delta \mathrm{q}}$ from experiments

- Dimensionality reduction by L1-regularization Resulting Mapping: $R_{\Delta \mathrm{q}}=\left(\begin{array}{c}0.312 \\ -0.722 \\ -78.4 \\ -0.216\end{array}\right) \cdot\left(\begin{array}{c}\boldsymbol{\mu}_{0}\left(\boldsymbol{t}_{\text {evap }}\right) \\ \boldsymbol{\mu}_{2}\left(\boldsymbol{v}_{\text {impact }}\right) \\ \boldsymbol{\mu}_{1}\left(\boldsymbol{x}_{\text {s,impact }}\right) \\ \boldsymbol{\mu}_{2}\left(\boldsymbol{x}_{\text {s,impact }}\right)\end{array}\right)+25.6$

Design of Experiments $\downarrow$ Result: Granulation Experiments Result:
Product Particles

Confocal Laser-Scanning Microscopy

$\downarrow$ Roughness Value

Map Roughness Values

to Tracked Quantities

Experimental Workflow

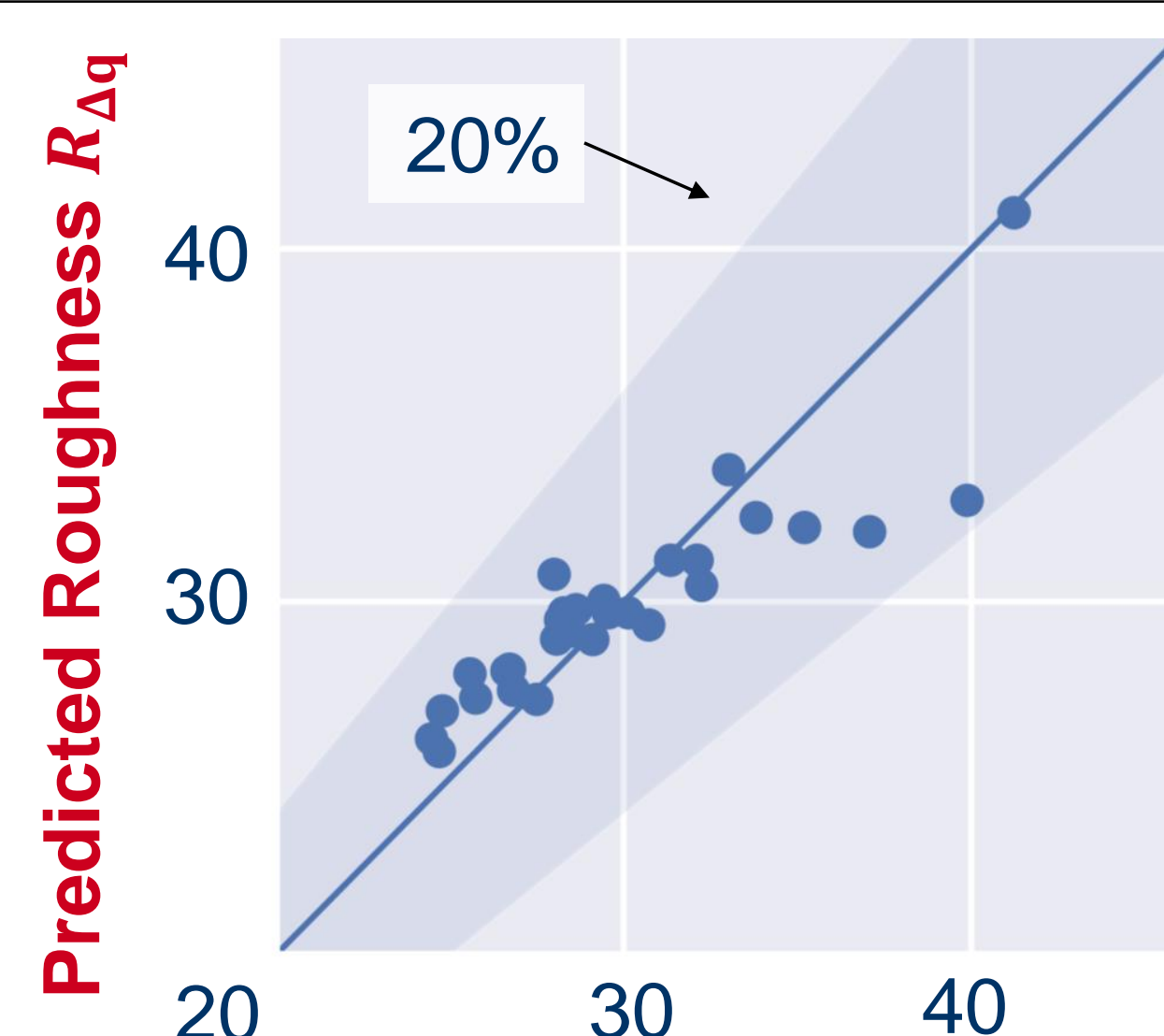

Measured Roughness $R_{\Delta}$ Parity Plot

\begin{tabular}{|c|c|c|c|c|}
\hline Summary & Variant & $\begin{array}{l}\text { Surface Liquid } \\
\text { Drying }\end{array}$ & $\begin{array}{l}\text { Droplet } \\
\text { Drying }\end{array}$ & $\begin{array}{l}\text { Droplet Impact } \\
\text { Velocity }\end{array}$ \\
\hline $\begin{array}{l}\text { CFD-DEM provides more information over } \\
\text { state-of-the-art design guidelines, } \\
\text { - uses distributions rather than } \\
\text { average quantities }\end{array}$ & $\begin{array}{l}\text { Stabilizing } \\
\text { Internals } \\
\text { (draft tube, } \\
\text { draft plates) }\end{array}$ & grjon & rime & (n) \\
\hline $\begin{array}{l}\text { - Advantage: Geometry-independence } \\
\text { Granulator geometries with identical } \\
\text { global drying conditions yield very } \\
\text { different tracked quantity distributions }\end{array}$ & $\begin{array}{l}\text { Counter- } \\
\text { Current } \\
\text { Spray } \\
\text { (vs bottom spray) }\end{array}$ & $\uparrow \uparrow \uparrow$ & $\uparrow \uparrow \uparrow$ & $\downarrow \downarrow \downarrow$ \\
\hline $\begin{array}{l}\text { - Largest influence: Direction of spray } \\
\text { Next Step: use method for scale-up }\end{array}$ & $\begin{array}{c}\text { Spouting } \\
\text { (vs bottom spray) }\end{array}$ & $\uparrow$ & $\uparrow \uparrow$ & $\downarrow$ \\
\hline
\end{tabular}

\section{Tracked Quantities in Different Granulators}



